\title{
Comment on Clinical Trial NCT00950001 and NCCTG/N107C/CEC.3: Are We Treating Cancer or Water?
}

\author{
Junfang Gao \\ Northeast Louisiana Cancer Institute, Monroe, LA, USA \\ Email: gao.junfang@yahoo.com
}

How to cite this paper: Gao, J.F. (2021) Comment on Clinical Trial NCT00950001 and NCCTG/N107C/CEC.3: Are We Treating Cancer or Water? International Journal of Medical Physics, Clinical Engineering and Radiation Oncology, 10, 12-15.

https://doi.org/10.4236/ijmpcero.2021.101002

Received: December 11, 2020

Accepted: January 31, 2021

Published: February 3, 2021

Copyright $\odot 2021$ by author(s) and Scientific Research Publishing Inc. This work is licensed under the Creative Commons Attribution International License (CC BY 4.0).

http://creativecommons.org/licenses/by/4.0/

\begin{abstract}
During the implementation of clinical trials NCT00950001 and NCCTG/ N107C/CEC.3 on post-operative stereotactic radiosurgery into clinic, it brought us some thinking of fundamental concept in science that the local control rate and survival rate rely on the treatment of marginal region more than resection cavity. Marginal region might still contain residual cancer cell while the resection cavity contains only water fluid most time. Radiation treatment should focus more on the margin rather than the cavity, thus treating cancer rather than water.
\end{abstract}

\section{Keywords}

NCT00950001, NCCTG/N107C/CEC.3

The publication of two clinical trials (NCT00950001 and NCCTG/N107C/CEC.3) [1] [2] in 2017, on post-operative stereotactic radiosurgery (SRS), has boosted SRS application in brain metastasis management. In the NCT00950001 trial, Dr. Mahajan and colleagues compared the medical endpoint between observation and adjuvant SRS therapy after neurosurgical resection of brain metastasis. In the NCCTG/N107C/CEC.3 trial, Dr. Brown and colleagues compared the medical outcome between SRS and WBRT (whole brain radiation therapy) among brain metastasis resection patients within 48 institutes. Even though some comments and criticisms have been addressed by other doctors [3] [4], these two informative clinical trials will potentially establish a standard guideline for clinicians in the treatment of post-operative brain metastasis patients. To better implement these trials into individual clinics and better care for individual patient, we need to look into some fundamental scientific concepts. Regarding the cavity 
delineation for SRS in these two trials, it caused me to think "Are we treating cancer or water?".

In a modern neurosurgical resection, craniotomy, craniectomy, debulking and gross total resection are the most common procedures performed on brain tumor patients either when the patient is awake or asleep under anesthesia [5]. After tumor resection, keeping the surgical cavity the same pressure gradient as the original brain, and avoiding cavity deformation due to motion and gravity are the major concerns of the neurosurgeon. Ordinary saline solution and artificial cerebrospinal fluid (CSF) are most often used in the cavity to maintain the pressure and formation. Sometimes a shunt has to be used to drain the excessive CSF to reduce the pressure build-up inside the cavity. Wafers are occasionally used as in-vivo chemotherapy inside the cavity. Overall, usually, the resection cavity filling substance is water. Since it was not especially addressed, the above two clinical randomized phase 3 trials are assumed to have managed the resection cavity following neurosurgeon standard practice. Most of the cavities in these trials are water substance occupied. In NCT00950001, only $1 \mathrm{~mm}$ margin is added outside the cavity to delineate the treatment volume (PTV) while in NCCTG/N107C/CEC. 3 only $2 \mathrm{~mm}$ margin is added outside the cavity to create the treatment volume (PTV). From the author's point of view, in these two trials, the majority of the radiation goes to the water inside the resection cavity while only very little radiation goes to the marginal region where most of the residual cancer cells are located. The residual cancer cells might also be located further than the $1 \mathrm{~mm}$ or $2 \mathrm{~mm}$ marginal region. That means these trials mainly focus on treating water rather than cancer.

The bulk water substance inside the cavity is different from the water molecules inside a tumor cell where water molecules can generate hydroxyl radicals to break the DNA after they absorb radiation. When the bulk water substance receives radiation from Gamma knife (average energy of $1.25 \mathrm{MeV}$ photon) or Linac (4 or $6 \mathrm{MeV}$ photon), the high energy photon interacts with bulk water inside the cavity mainly through three processes. They are photoelectric $(\mathrm{E}<0.1$ $\mathrm{MeV})$, Compton scattering $(\mathrm{E}<10 \mathrm{MeV})$ and electron-positron pair production $(\mathrm{E}>1.02 \mathrm{MeV})[6]$. These are ionization processes and create positive and negative charge counterparts. In bulk water substance, the positive and negative charge exists for a very short time period and then combines into a new water molecule again. At the end of radiation, the water amount remains the same. This can be very easily seen in water phantom (inside water tank) based Linac commissioning and annual and monthly Linac QA by the physicist. The Physicist delivers millions of Gy radiation dose to the water phantom and the water never shrinks or disappears (except some natural evaporation). All this water has the nature of "being resurrected" in big bulk tank during radiation. This analogy can be applied to the SRS water cavity irradiation in both NCT00950001 and N107C/CEC.3 trials.

Figure 1 is the one image we frequently see in SRS planning in clinic. Red 


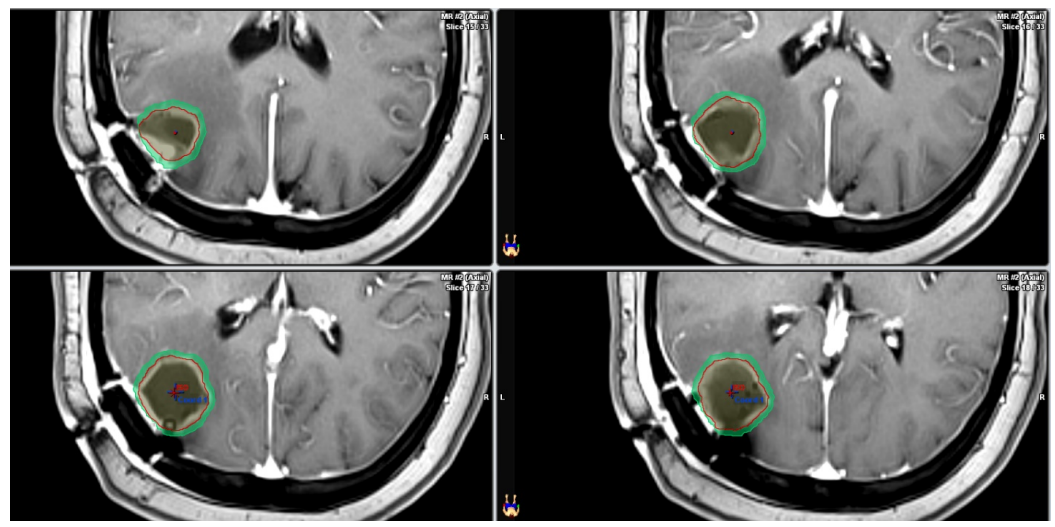

Figure 1. Axial view of one set T1 with contrast MRI image for post-op stereotactic radiosurgery. Red contour is GTV and Green contour is PTV which is $2 \mathrm{~mm}$ expansion from GTV.

contour represents GTV and green contour represents PTV, which is $2 \mathrm{~mm}$ expansion from GTV following NCCTG/N107C/CEC.3 clinical trial. The surgical cavity is in black and full of water substance which will be the target to receive majority radiation.

Therefore, when the authors design those trials, they should focus more on treating the marginal region rather than cavity. The systemic study of marginal region treatment will make more sense in the investigation of the local control rate and survival rate. I would propose the recommendation to other clinicians that after establishing a proper margin based on post-op cavity, treatment should be focused more on the marginal region rather than the cavity, thus treating cancer rather than water.

\section{Conflicts of Interest}

The author declares no conflicts of interest regarding the publication of this paper.

\section{References}

[1] Mahajan, A., Ahmed, S., McAleer, M.F., et al. (2017) Post-Operative Stereotactic Radiosurgery versus Observation for Completely Resected Brain Metastases: A Single-Centre, Randomised, Controlled, Phase 3 Trial. The Lancet Oncology, 18, 1040-1048. https://doi.org/10.1016/S1470-2045(17)30414-X

[2] Brown, P.D., Ballman, K.V., Cerhan, J.H., et al. (2017) Postoperative Stereotactic Radiosurgery Compared with Whole Brain Radiotherapy for Resected Metastatic Brain Disease (NCCTG N107C/CEC.3): A Multicentre, Randomised, Controlled, Phase 3 Trial. The Lancet Oncology, 18, 1049-1060.

https://doi.org/10.1016/S1470-2045(17)30441-2

[3] Lo, S.S., Chang, E.L. and Sahgal, A. (2017) Radiosurgery for Resected Brain Metastases: A New Standard of Care? The Lancet Oncology, 18, 985-987. https://doi.org/10.1016/S1470-2045(17)30448-5

[4] Yue, J.B. and Yu, J.M. (2017) SRS versus WBRT for Resected Brain Metastases. The Lancet Oncology, 18, e559. https://doi.org/10.1016/S1470-2045(17)30642-3 
[5] https://www.abta.org/wp-content/uploads/2019/07/Surgery Brochure 2020 web e $\underline{\text { n.pdf }}$

[6] Khan, F.M. (2010) Chapter 5: The Physics of Radiation Therapy. Fourth Edition, 54-69. 